\title{
Deguelin induces apoptosis in colorectal cancer cells by activating the p38 MAPK pathway
}

This article was published in the following Dove Press journal:

Cancer Management and Research

\author{
Liubo Chen ${ }^{1,2, *}$ \\ Kai Jiang ${ }^{1,2, *}$ \\ Haiyan Chen ${ }^{1,3, *}$ \\ Yang Tang ${ }^{1,2}$ \\ Xinyi Zhou ${ }^{1,2}$ \\ Yinuo $\operatorname{Tan}^{1,4}$ \\ Ying Yuan ${ }^{1,4}$ \\ Qian Xiao ${ }^{1,2}$ \\ Kefeng Ding ${ }^{1,2}$ \\ 'Cancer Institute (Key Laboratory of \\ Cancer Prevention and Intervention, \\ China National Ministry of Education, \\ Key Laboratory of Molecular \\ Biology in Medical Sciences, Zhejiang \\ Province, China), The Second Affiliated \\ Hospital, Zhejiang University School \\ of Medicine, Hangzhou, Zhejiang, \\ China; ${ }^{2}$ Department of Surgical \\ Oncology, The Second Affiliated \\ Hospital, Zhejiang University School \\ of Medicine, Hangzhou, Zhejiang, \\ China; ${ }^{3}$ Department of Radiation \\ Oncology, The Second Affiliated \\ Hospital, Zhejiang University School \\ of Medicine, Hangzhou, Zhejiang, \\ China; ${ }^{4}$ Department of Medical \\ Oncology, The Second Affiliated \\ Hospital, Zhejiang University School \\ of Medicine, Hangzhou, Zhejiang, \\ China
}

*These authors contributed equally to this work

Correspondence: Kefeng Ding; Qian Xiao Department of Surgical Oncology, The Second Affiliated Hospital, Zhejiang University School of Medicine, 88 jiefang Road, Hangzhou, Zhejiang 310009, China Tel +86 57| 87784760

Fax $+8657 \mid 87783458$

Email dingkefeng@zju.edu.cn; qxiao3@ zju.edu.cn
Objectives: Deguelin, a rotenoid extracted from Mundulea sericea (Leguminosae), exhibits antitumor effects on several types of human cancers. Due to the limited studies of deguelin on colorectal cancer (CRC), the present study was designed to investigate the antitumor effect of deguelin and to explore the underlying mechanism in CRC.

Materials and methods: Cell viability was assessed by the cell counting kit-8 (CCK-8) assay, and cell apoptosis was determined by the annexin v-propidium iodide staining using flow cytometry and Western blot in CRC cell lines after incubation with deguelin. The antitumor effect of deguelin was further evaluated in tumor xenograft models. Moreover, SB203580, a specific inhibitor of p38 MAPK, was used to confirm the involvement of 38 MAPK pathway in deguelin-induced apoptosis. Results: Deguelin significantly inhibited cell proliferation and induced apoptosis in CRC cell lines (SW620 and RKO) in a time-dependent and dose-dependent manner. Western blot analysis also showed that the expression of proapoptotic proteins (cleaved caspase 3 and cleaved PARP) was upregulated, while that of antiapoptotic proteins (Bcl-2 and survivin) was downregulated after deguelin treatment in CRC cell lines. Moreover, oral administration of deguelin significantly suppressed tumor growth and induced apoptosis in subcutaneous xenograft mouse models without obvious toxicity. Additionally, Western blot revealed that deguelin-induced apoptosis might be regulated by the 338 MAPK pathway and inhibition of p38 MAPK could attenuate deguelin-induced proliferative inhibition and apoptosis in CRC cells.

Conclusion: Collectively, these results demonstrated that deguelin inhibited CRC cell growth by inducing apoptosis via activation of 338 MAPK pathway.

Keywords: deguelin, colorectal cancer, apoptosis, p38 MAPK pathway

\section{Introduction}

Colorectal cancer (CRC) is among the leading causes of cancer-related mortality in Western countries ${ }^{1}$ and the fifth most common cause of cancer death in China. ${ }^{2}$ The prognosis of advanced CRC is poor, and the current regimens mainly consist of surgical resection, chemoradiotherapy, and targeted therapy. ${ }^{3}$ In spite of advances in cytotoxic chemotherapy in recent decades, the severe side effects and chemoresistance remain a global challenge. The introduction of targeted therapy to metastatic CRC, including anti-EGFR and anti-VEGFR, has improved the patient survival to some extent. Nonetheless, identifying novel alternative therapy is an encouraging approach to complement the current anticancer therapy.

Deguelin, a rotenoid extracted from Mundulea sericea (Leguminosae), has been found to exhibit chemopreventive and therapeutic activities in several types of cancers. $^{4-7}$ Lee et $\mathrm{al}^{5,8}$ have found that deguelin exerts its chemopreventive effects 
in tobacco-induced lung tumorigenesis by decreasing the expression of cyclooxygenase-2. In premalignant and malignant human bronchial epithelial cells, PI3K/Akt pathway is suppressed following deguelin administration, thereby inhibiting cell proliferation and inducing apoptosis. ${ }^{9}$ In addition, deguelin has been demonstrated to induce apoptosis in breast cancer and lung cancer by targeting the Wnt pathway and by inhibiting the expression of galectin-1, respectively. ${ }^{4,6}$ It has also been reported that deguelin is an active antiangiogenic agent by targeting hypoxia-inducible factor- $1 \alpha .{ }^{10}$ In triplenegative MDA-MB-231 breast cancer cell lines, deguelin inhibits the insulin-like growth factor-1 receptor (IGF-1R) signaling activation mainly by upregulating IGF-binding protein-3 expression, thereby exerting an anticancer activity. ${ }^{11}$ Speculated from above-described characteristics, deguelin may be a potential chemotherapeutic agent in several kinds of malignancies, including CRC. However, studies concerning the antitumor effect of deguelin in $\mathrm{CRC}$ are limited. Although deguelin has been revealed to downregulate NF- $\kappa \mathrm{B}$ signaling and induce apoptosis in COLO 205 and HCT116 cells, ${ }^{12}$ the mechanisms remain poorly understood.

In this study, we evaluated the effect of deguelin in CRC cells in vitro and in vivo. The data showed that deguelin inhibited cell proliferation in a time-dependent manner and dose-dependent manner. Flow cytometry and Western blot analyses revealed significant apoptosis in CRC cell lines (SW620 and RKO) following deguelin treatment. Consistently, in xenograft mouse model, deguelin suppressed tumor growth and induced apoptosis without obvious toxicity. Moreover, the activation of $\mathrm{p} 38$ MAPK pathway was involved in deguelin-induced apoptosis. And inhibition of p38 MAPK pathway by SB203580, a specific inhibitor of p38 MAPK, attenuated deguelin-induced apoptosis.

\section{Materials and methods}

\section{Chemicals and reagents}

Deguelin was purchased from Sigma-Aldrich Co. (St Louis, MO, USA). SB203580, an inhibitor of p38 MAPK, was obtained from Selleck Chemicals Company (Houston, TX, USA). Both of the chemicals were dissolved in dimethylsulfoxide (DMSO) (Sigma-Aldrich Co.) and stored at $-20^{\circ} \mathrm{C}$. Cottonseed oil was purchased from Sigma-Aldrich Co.

\section{Cell culture}

Human CRC cell lines (SW480, SW620, and RKO) were obtained from the American Type Culture Collection (ATCC) (Manassas, VA, USA). All cells were maintained in RPMI 1640 medium (Thermo Fisher Scientific, Waltham, MA,
USA), supplemented with 10\% fetal bovine serum (Thermo Fisher Scientific), $100 \mathrm{U} / \mathrm{mL}$ of penicillin, and $100 \mathrm{mg} / \mathrm{mL}$ of streptomycin at $37^{\circ} \mathrm{C}$ in a humidified atmosphere of $5 \% \mathrm{CO}_{2}$.

\section{Cell viability analysis}

Cell viability was analyzed using the cell counting kit-8 (CCK-8) (Dojindo Laboratories, Tokyo, Japan). Briefly, cells were seeded into 96 -well plates at a density of 5-10 $\times 10^{3}$ cells/ well for overnight. The working solution of deguelin was diluted with RPMI 1640 medium with a maximal concentration of DMSO at $0.1 \%$. Cells were treated with indicated concentrations of deguelin for 6, 12, 24, and 48 hours. After incubation, CCK-8 was added into each well. The absorbance was measured using a microplate reader at $450 \mathrm{~nm}$ after incubation for additional 3 hours. Three replicate wells were set up in each group.

\section{Measurement of apoptosis by annexin $\mathrm{V}$-propidium iodide $(\mathrm{PI})$ assay}

Measurement of cell apoptosis was determined by Annexin V-FITC Apoptosis Detection Kit (Dojindo Laboratories) according to the manufacturer's instructions. In brief, after deguelin treatment, cells were harvested and washed with PBS twice. Cells were then resuspended in binding solution (containing $5 \mu \mathrm{L}$ of annexin V-FITC and $5 \mu \mathrm{L}$ of PI), followed by incubation at room temperature in darkness for 15 minutes. Analysis was carried out within 1 hour using the flow cytometer (FACSCanto II; BD Biosciences, San Jose, CA, USA).

\section{Mouse xenograft CRC model}

The animal experiments were approved by the Animal Care and Use Committee of the Second Affiliated Hospital of Zhejiang University School of Medicine. And, we strictly performed the animal experiments according to the protocols approved by the Animal Care and Use Committee of the Second Affiliated Hospital of Zhejiang University School of Medicine.

Five to six-week-old female nude mice were purchased from SLAC Laboratory Animal Co. Ltd. (Shanghai, China). SW620 cells $\left(1 \times 10^{6}\right)$ were suspended in PBS and injected subcutaneously into mice. Tumor growth was monitored daily until the tumor was palpable $\left(50-100 \mathrm{~mm}^{3}\right)$. Then, the mice were randomized into two groups and were administered with vehicle (DMSO) or deguelin ( $4 \mathrm{mg} / \mathrm{kg}$ ) suspended in $0.1 \mathrm{~mL}$ of cottonseed oil daily by oral gavage. ${ }^{11,13}$ Body weight and tumor size were measured every 3 days. Tumor volumes were calculated using the following formula: tumor volume $\left(\mathrm{mm}^{3}\right)=L \times S \times S / 2$, where $L$ is the long axis of tumor and $S$ represents the short axis 
of tumor. After 24 days of deguelin administration, mice were sacrificed and tumor was excised, photographed, and further fixed in $10 \%$ neutral formalin, embedded in paraffin.

\section{TUNEL assay}

The TUNEL assay was performed using the In Situ Cell Death Detection Kit (Hoffman-La Roche Ltd., Basel, Switzerland) according to the manufacturer's instruction. Briefly, the paraffin-embedded sections were deparaffinized in xylene and dehydrated in a graded series of ethanol. Then, the slides were rinsed in PBS and incubated with Proteinase $\mathrm{K}$ for 20 minutes at room temperature. After washing with PBS, the sections were reacted with TUNEL solution in dark for 1 hour at room temperature. Afterward, converter-peroxidase solution was added and incubated in dark for 30 minutes at room temperature, followed by incubation with DAB for 10 minutes at room temperature and subsequent hematoxylin counterstaining. Representative images were photographed at $10 \times$ or $40 \times$ objective ( 100 or $400 \times$ magnification) under microscope (Olympus Corporation, Tokyo, Japan).

\section{Western blot assay}

Total protein was extracted from SW620 and RKO cells after deguelin treatment, followed by the determination of protein concentration by Pierce BCA Protein Assay Kit (Thermo Fisher Scientific). Protein samples were subjected to $10-12 \%$ SDS-PAGE and transferred to polyvinylidene fluoride (PVDF) membrane (Bio-Rad Laboratories Inc., Hercules, CA, USA). After blocking with 5\% nonfat milk for 1 hour at room temperature, the membranes were incubated with appropriate primary antibodies overnight at $4{ }^{\circ} \mathrm{C}$. Primary antibodies against total caspase 3, cleaved caspase 3, PARP, cleaved PARP, Bcl-2, survivin, phosphorylated-JNK (Thr183/ Tyr185), phosphorylated-ERK1/2, and phosphorylated-p38 MAPK (Thr180/Tyr182) were purchased from Cell Signaling Technology (CST, Boston, MA, USA) (all dilution 1:1,000). Anti-p38 MAPK and anti-NF-אB p65 (all dilution 1:2,000) were purchased from Abcam (Cambridge, MA, USA). A mouse anti- $\beta$-actin monoclonal antibody (dilution 1:1,000; CST) was used as a loading control. Following incubation with secondary antibody conjugated with horseradish peroxidase for 1 hour at room temperature, the immunoreactive bands were visualized using the enhanced chemiluminescence (ECL) detection system (Thermo Fisher Scientific).

\section{Statistical analysis}

All data were presented as mean \pm standard error of the mean (SEM) of three independent experiments. Statistical analysis was performed by Student's $t$-test or ANOVA multiple comparisons in GraphPad Prism Version 6.0 software (GraphPad Software, Inc., La Jolla, CA, USA). Differences were considered significant at $* P<0.05, * * P<0.01$, and $* * * P<0.001$.

\section{Results \\ Deguelin inhibited cell viability in CRC cells}

To evaluate the effects of deguelin on CRC cell viability, SW480 and SW620 cells were treated with 0-200 nM deguelin for 24 and 48 hours. As shown in Figure 1A and $\mathrm{B}$, the $50 \%$ inhibitory concentrations $\left(\mathrm{IC}_{50}\right)$ of deguelin for 24 hours in SW480 and SW620 cells were 122.02 and $36.57 \mathrm{nM}$, respectively. After incubation for 48 hours, the $\mathrm{IC}_{50}$ of deguelin in SW480 and SW620 cells were 40.86 and $17.73 \mathrm{nM}$, respectively. Similarly, RKO cells were incubated with $0-20 \mu \mathrm{M}$ deguelin for 24 and 48 hours, with the $\mathrm{IC}_{50}$ for 48 hours being $13.25 \mu \mathrm{M}$ (Figure 1C). Furthermore, SW480 and SW620 cells were incubated with deguelin at $100 \mathrm{nM}$, while RKO cells were administered with $10 \mu \mathrm{M}$ deguelin for 6, 12, 24, and 48 hours. As shown in Figure 1D-F, deguelin started to exert a significantly inhibitory effect on SW480 and RKO cells after incubation for 12 hours. Notably, cell proliferation of SW620 was significantly suppressed after exposure to deguelin for 6 hours. Together, deguelin inhibited CRC cell proliferation in a dose-dependent and timedependent manner.

\section{Deguelin induced apoptosis in CRC cells}

To determine whether deguelin-inhibited cell viability was induced by apoptosis, annexin V-FITC/PI staining using flow cytometry was performed. As shown in Figures 2A and B and $\mathrm{S} 1 \mathrm{~A}$ and $\mathrm{B}$, incubation with $50 \mathrm{nM}$ deguelin for 24 hours induced approximately $30 \%$ of apoptotic rate in SW620 cells, which rose to over $90 \%$ of apoptotic rate after incubation for 48 hours. As for RKO cells, high doses of deguelin (up to $10 \mu \mathrm{M}$ ) induced significant apoptosis (Figures 2C and D and S1C and D). Collectively, deguelin induced apoptosis in SW620 and RKO cells in a dose-dependent and timedependent pattern.

\section{Deguelin inhibited tumor growth and induced apoptosis in the xenograft mouse model}

To confirm our findings, we assessed the antitumor effect of deguelin in vivo. SW620 cells were injected into the flanks of nude mice. After the palpable tumors reached $50-100 \mathrm{~mm}^{3}$, 
A

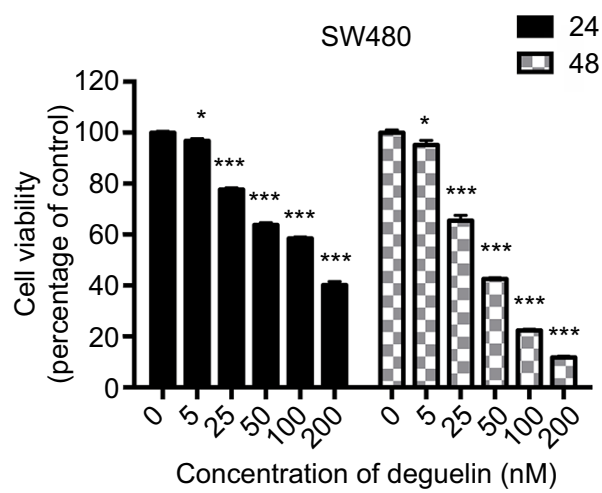

C

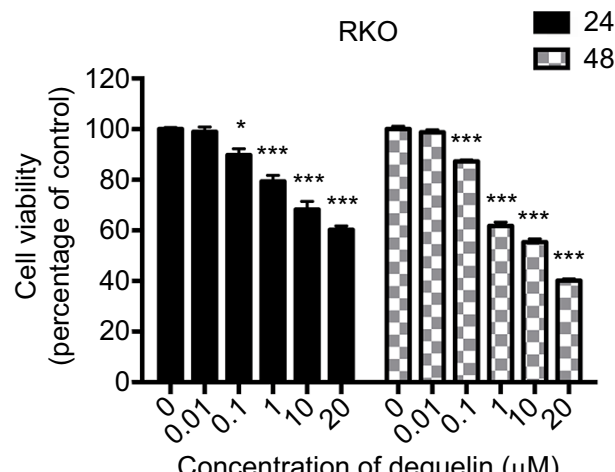

E

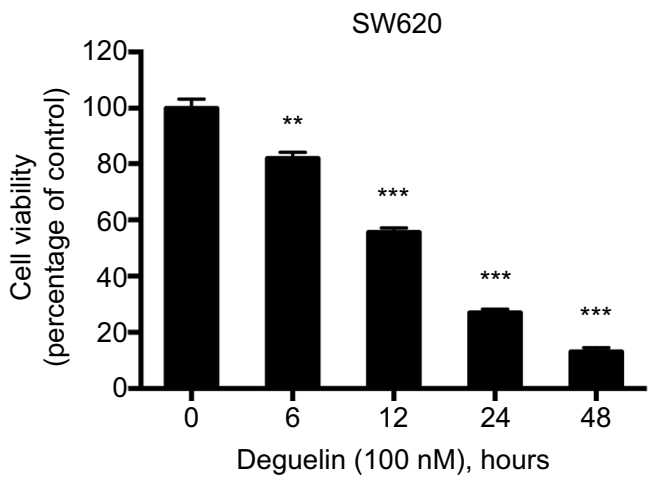

24 hours

48 hours
24 hours 48 hours

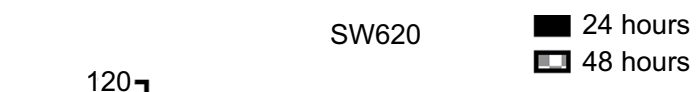

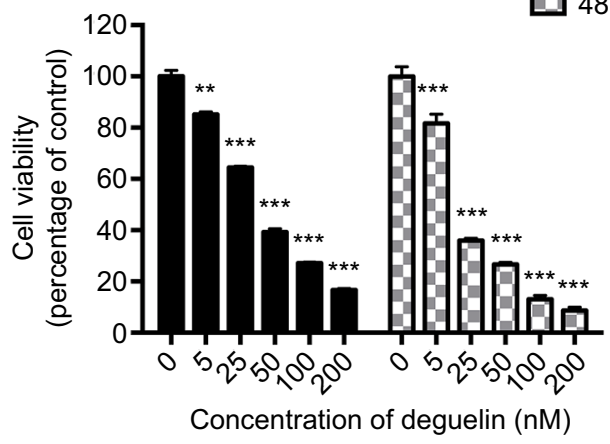

D

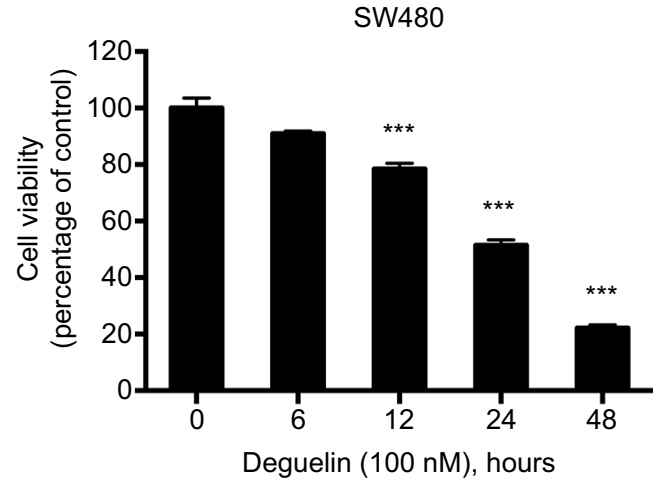

F

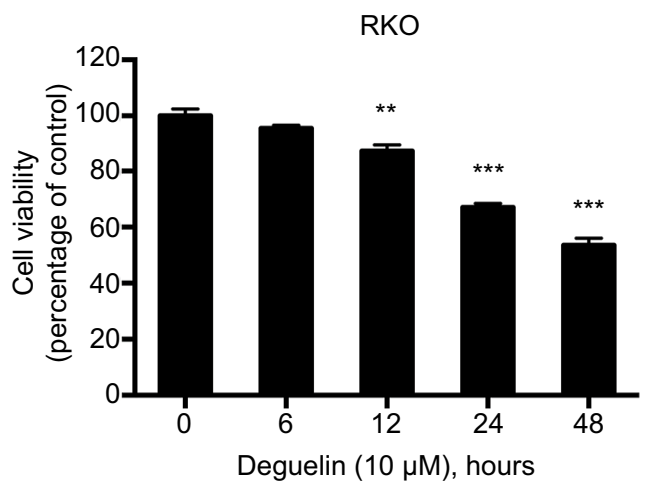

Figure I Deguelin inhibited proliferation of human colorectal cancer cell lines in a dose- and time-dependent manner.

Notes: Cell viability was determined using the CCK-8 assay. (A) SW480, (B) SW620, and (C) RKO cells were treated without or with different concentrations of deguelin for 24 and 48 hours. The IC $\mathrm{I}_{50}$ of deguelin for 24 hours in SW480 and SW620 were $122.02 \mathrm{nM}$ and 36.57 nM, respectively. And the IC 50 of deguelin for 48 hours in SW480, SW620, and RKO cells were $40.86 \mathrm{nM}, 17.73 \mathrm{nM}$, and I3.25 MM, respectively. (D) SW480 and (E) SW620 cells were cultured with deguelin (I00 nM) for different time points. (F) RKO cells were cultured with deguelin $(10 \mu \mathrm{M})$ for different time points. The data are presented as mean \pm SEM for three different experiments performed in triplicate. $* P<0.05$, $* * P<0.01$ and $* * * P<0.001$, compared to control group.

Abbreviations: CCK-8, cell counting kit-8; $I_{50}, 50 \%$ inhibitory concentrations; SEM, standard error of the mean. 
A

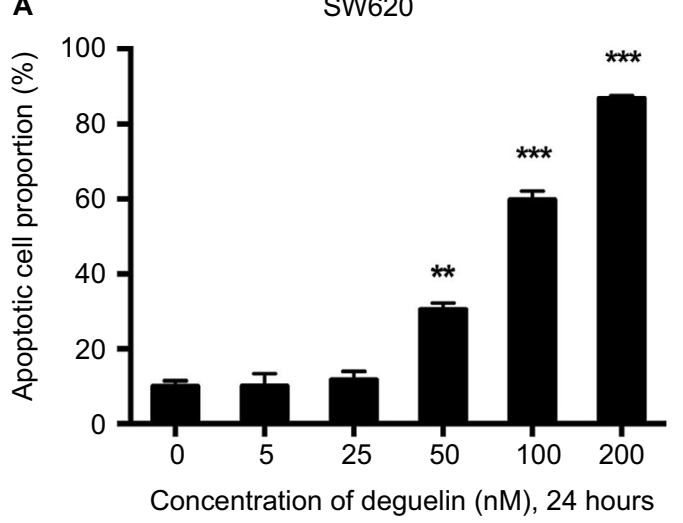

C

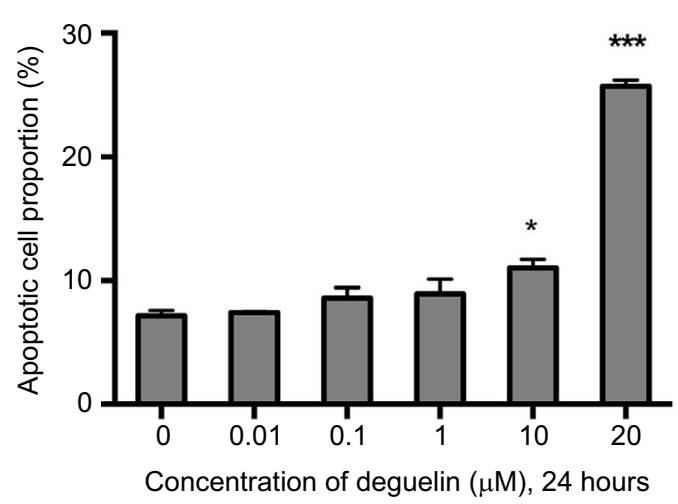

B

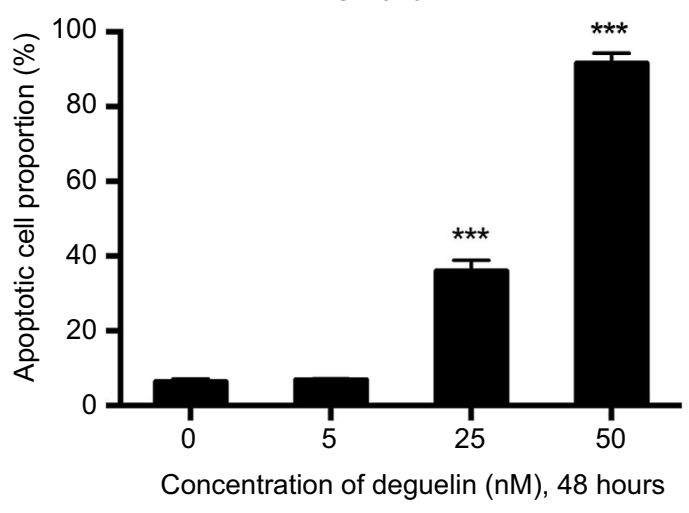

D

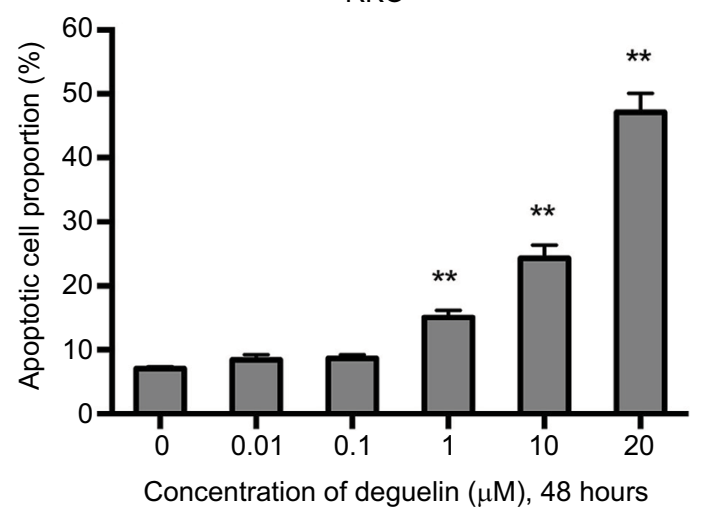

Figure 2 Deguelin induced apoptosis in human colorectal cancer cell lines.

Notes: Cell apoptosis was evaluated by flow cytometry after annexin V and PI staining as described in the "Materials and methods" section. SW620 cells were treated without or with different concentrations of deguelin for (A) 24 hours or (B) 48 hours. RKO cells were treated without or with different concentrations of deguelin for (C) 24 hours or (D) 48 hours. The data are presented as the mean \pm SEM of three independent experiments. $* P<0.05$, $* * P<0.0$ I and $* * * P<0.00$ I, compared to control group. Abbreviation: SEM, standard error of the mean.

nude mice were randomly assigned to receive either vehicle or deguelin by oral gavage ( $\mathrm{N}=6$ in each group). As shown in Figure 3A, deguelin treatment had little effect on body weight, demonstrating the low toxicity of deguelin. A marked inhibition of tumor growth was observed in mice receiving oral deguelin treatment, as indicated by reduced tumor size compared to the vehicle-treated group (Figure 3B). At the terminal of the experiment, mice were sacrificed and the tumors were excised and weighed. The average tumor weight was $1.485 \pm 0.255 \mathrm{~g}$ in vehicle-treated group, compared with $0.702 \pm 0.134 \mathrm{~g}$ in the deguelin-treated group $(P<0.001)$ (Figure 3C and D). TUNEL assay is widely adopted in apoptosis detection in tissue sections. ${ }^{14}$ Here, in our study, TUNEL assay demonstrated stronger staining in tumor tissues from deguelin-treated mice compared to the control group (Figure $3 \mathrm{E}$ ), indicating a higher apoptotic rate in the deguelin-administered mice. Taken together, these results indicated that deguelin inhibited tumor growth and induced apoptosis in vivo.

\section{Deguelin upregulated apoptotic protein expressions and downregulated antiapoptotic protein levels in CRC cells}

To confirm the effect of deguelin on cell apoptosis, we tested the apoptotic and antiapoptotic protein expressions after deguelin treatment. Western blot revealed that incubation with deguelin for 24 hours at doses 10 and $20 \mu \mathrm{M}$ increased the accumulation of cleaved-caspase 3 and cleaved-PARP while decreased the expression of Bcl-2 and survivin (antiapoptotic genes) in RKO cells (Figure 4A). Consistently, the expression of antiapoptotic proteins (Bcl-2 and survivin) was downregulated in SW620 cells 
A

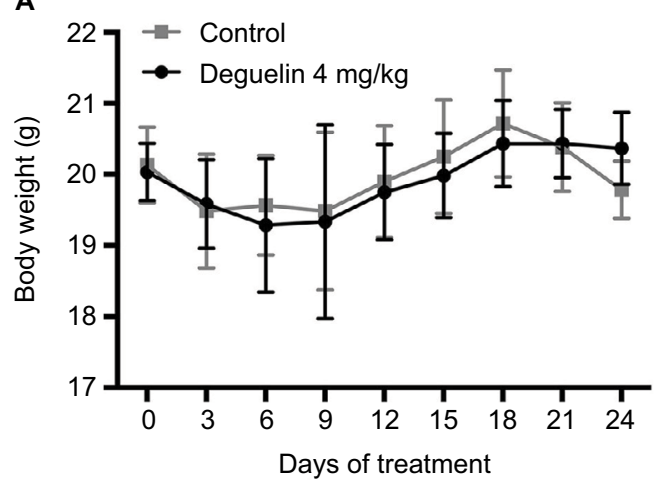

C

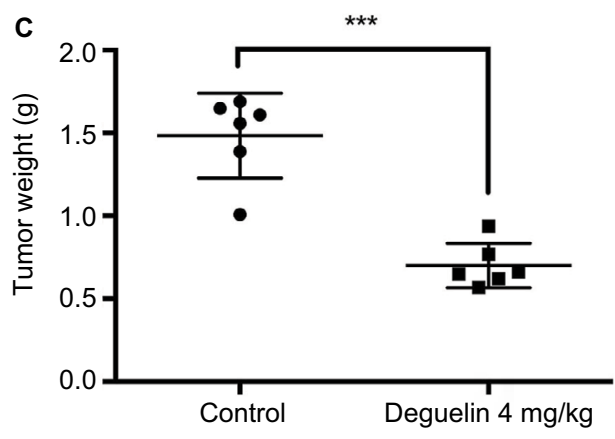

E

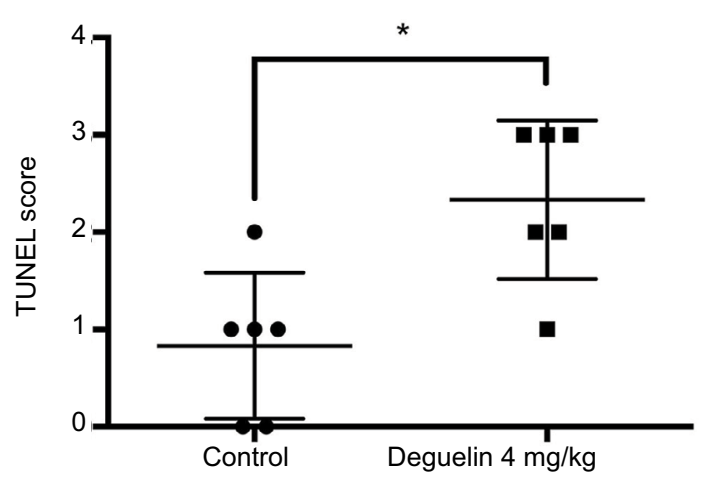

B

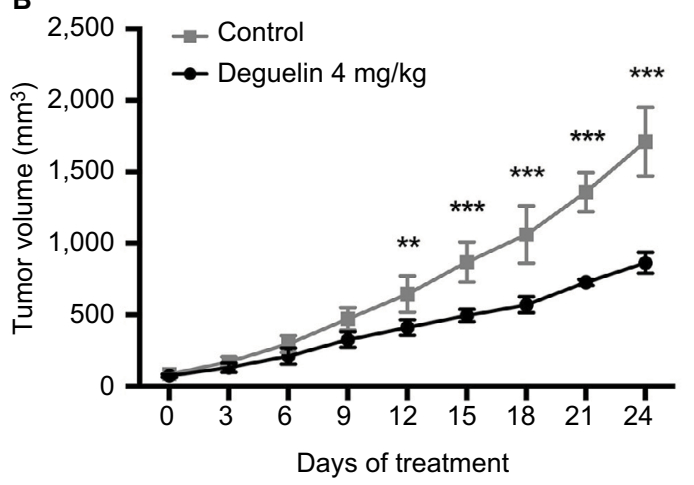

D
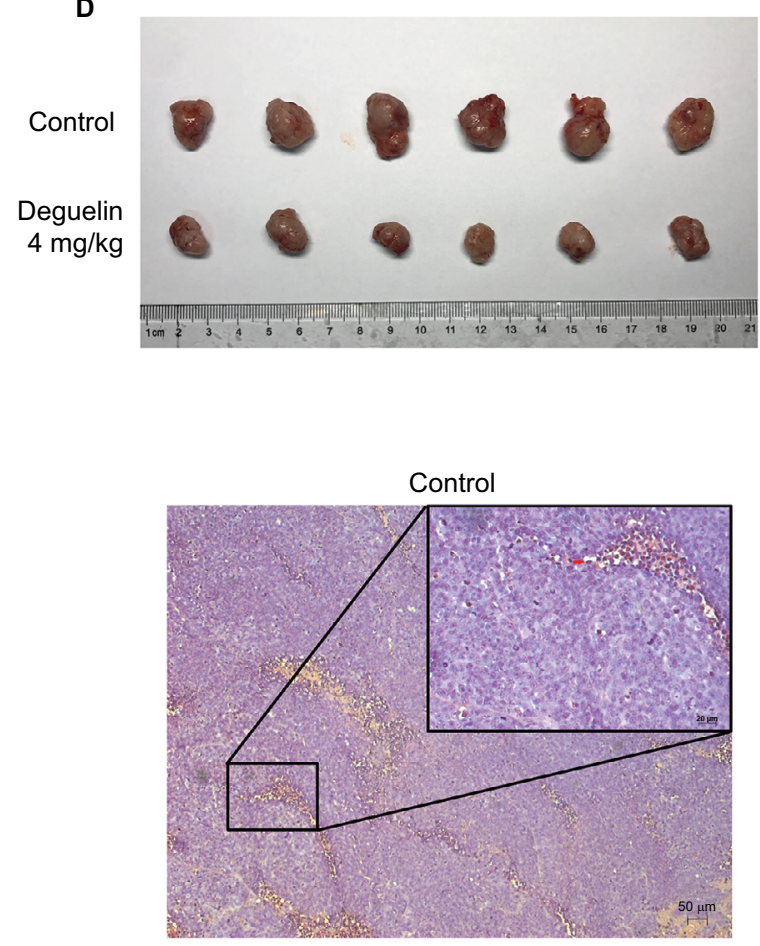

Deguelin 4 mg/kg

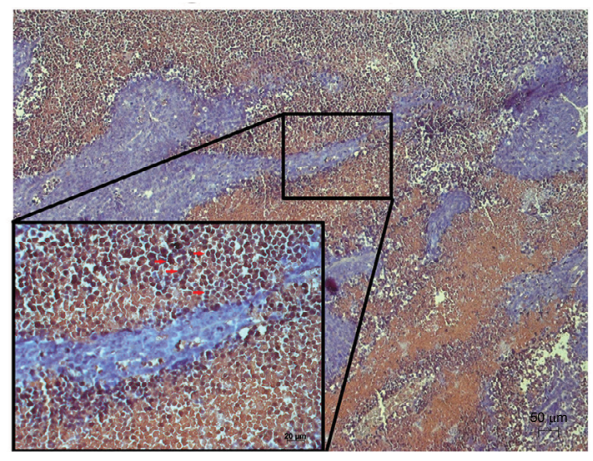

Figure 3 Deguelin inhibited tumor growth in the xenograft mouse model.

Notes: (A) Deguelin administration had no significant effect on body weight. (B) Tumor volumes were measured every 3 days after the palpable tumors reached 50$100 \mathrm{~mm}^{3}$. Tumor growth was significantly inhibited after exposure to deguelin for 12 days compared to the control group. (C) Tumor weight was assessed on sacrificed mice, suggesting a significantly decreased tumor weight in mice treated with deguelin. (D) Representative images of gross morphology. (E) TUNEL assay revealed a higher apoptotic rate in deguelin-treated mice ( $N=6$ for each group). $* P<0.05, * * P<0.01$ and $* * * P<0.00 \mathrm{I}$, compared to control group. 
A

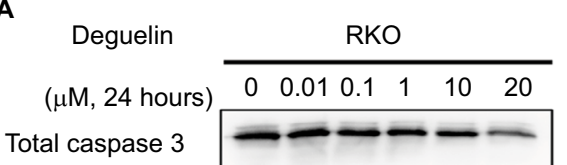

Cleaved caspase $3 \longdiv { - \square }$

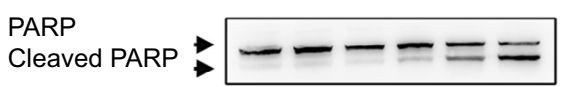

Bcl-2

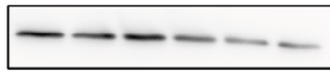

Survivin

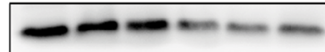

$\beta$-Actin

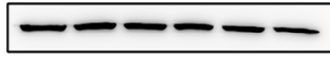

C

Deguelin

\begin{tabular}{lllllll}
\cline { 2 - 5 }$(\mu \mathrm{M}, 24$ hours $)$ & 0 & 0.01 & 0.1 & 1 & 10 & 20
\end{tabular}

p-JNK

$---=-$

p-Erk1/2

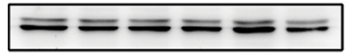

Total p38 MAPK

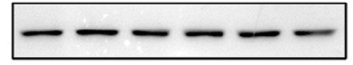

p-p38 MAPK

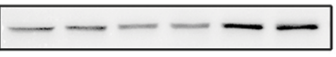

$N F-\kappa B p 65$

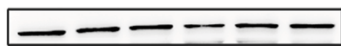

$\beta$-Actin

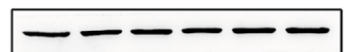

E

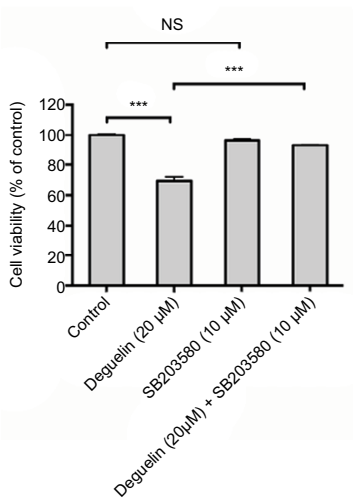

$\mathbf{F}$

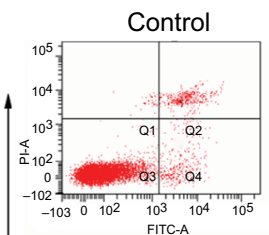

$\bar{\alpha}$

Deguelin $(20 \mu \mathrm{M})+$$$
10^{5}
$$

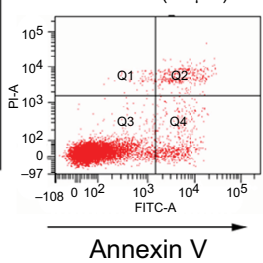

B

Deguelin

(nM, 24 hours)

Total caspase 3

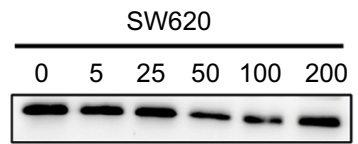

Cleaved caspase 3

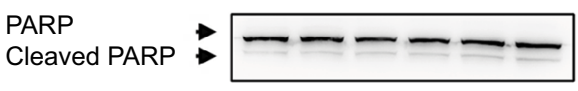

$\mathrm{Bcl}-2$

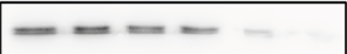

Survivin

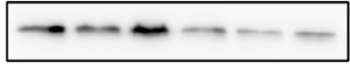

$\beta$-Actin

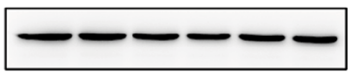

D
$\beta$-Actin

SB203580 $(10 \mu \mathrm{M})-\quad+\quad+$

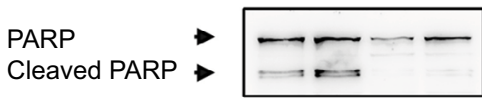

Cleaved caspase 3

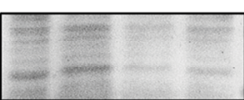

p-p38 MAPK
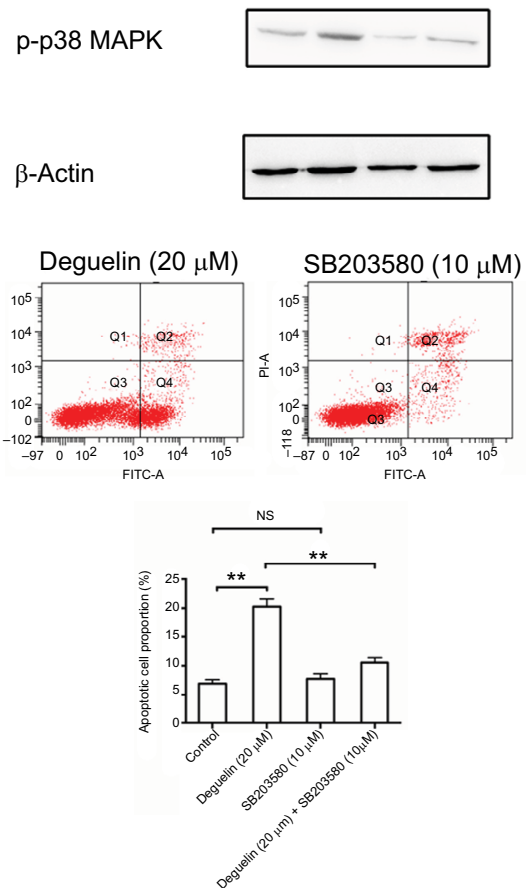

Figure 4 Deguelin induced apoptosis in colorectal cancer cells via activation of $\mathrm{p} 38$ MAPK pathway.

Notes: (A) RKO and (B) SW620 cells were treated with indicated concentrations of deguelin for 24 hours. The total lysates were subjected to Western blot analysis, and $\beta$-actin was used as an internal reference. (C) Analysis of MAPK and NF- $\kappa B$ pathways after deguelin treatment. As a result, phosphorylated form of $\mathrm{p} 38$ was upregulated, while no obvious changes were detected in P-JNK, p-ERKI/2, or p65. Administration of SB203580 at a dose of $10 \mu M$, a specific inhibitor of p38 MAPK, could reverse deguelininduced (E) cell proliferation inhibition and (D and F) apoptosis in RKO cells. Briefly, SB203580 was dissolved in DMSO at 10 mM as stocking solution, which was further diluted into $10 \mu \mathrm{M}$ as working solution. $* * P<0.01$ and $* * * P<0.001$, compared to control group.

Abbreviations: DMSO, dimethylsulfoxide; NS, not significant. 
following deguelin administration, although deguelin treatment did not lead to obvious cleavage of caspase 3 (Figure 4B).

\section{Deguelin induced apoptosis in CRC cells by activating the p38 MAPK pathway}

In consideration of the critical role of MAPK and NF- $\kappa B$ signaling in the regulation of cell apoptosis, ${ }^{15-17}$ we evaluated the activity of NF- $\mathrm{B}$ and MAPK pathway after deguelin treatment. As shown in Figure $4 \mathrm{C}$, phosphorylated form of p38 MAPK increased after deguelin administration for 24 hours, whereas no significant changes were observed in phosphorylated-Erk1/2, phosphorylated-JNK, or NF- $\mathrm{B}$ $\mathrm{p} 65$. Moreover, to further confirm the involvement of $\mathrm{p} 38$ MAPK in deguelin-induced apoptosis, RKO cells were pretreated with $10 \mu \mathrm{M}$ SB203580, a specific inhibitor of p38 MAPK. Afterward, deguelin $(20 \mu \mathrm{M})$ was added to the pretreated cells and incubated for another 24 hours. As a result, SB203580 decreased the expression of phosphorylated-p38 MAPK (Figure 4D). CCK-8 assay revealed that the suppression of $\mathrm{p} 38 \mathrm{MAPK}$ attenuated deguelin-triggered inhibition of cell proliferation (Figure 4E). Consistently, inhibition of p38 MAPK led to attenuated apoptosis, as indicated by flow cytometry and Western blot (Figure 4F and D). Therefore, activation of the p38 MAPK pathway was involved in deguelin-induced apoptosis in CRC cells and inhibition of the p38 MAPK pathway could attenuate deguelin-triggered apoptosis.

\section{Discussion}

In this study, we reported that deguelin inhibited cell proliferation and induced apoptosis in a time-dependent and dose-dependent manner in CRC cells. In xenograft mouse model, both tumor sizes and tumor weights were significantly suppressed without obvious toxicity following deguelin administration. TUNEL assay revealed a more obvious apoptosis in tumors excised from mice receiving oral deguelin treatment compared to those from the control group. Inhibition of p38 MAPK could attenuate deguelininduced apoptosis in CRC cells. To the best of our knowledge, we demonstrated for the first time that deguelin induced apoptosis in CRC cells partially via activation of p38 MAPK pathway.

In the evaluation of the cytotoxicity of deguelin, we found that the $\mathrm{IC}_{50}$ values for SW620, SW480, and RKO varied. Obvious growth inhibition was observed at the nanomole of deguelin in SW620 and SW480 cells, while micromole of deguelin was necessary to suppress the proliferation of RKO cells. By referring to previous studies, we found that the $\mathrm{IC}_{50}$ (deguelin, 24 hours) of PanC-1, a pancreatic adenocarcinoma cell line, was $62 \mu \mathrm{M},{ }^{7}$ while the $\mathrm{IC}_{50}$ (deguelin, 24 hours) of H60, a human lung cancer cell line, was only around $250 \mathrm{nM} \cdot{ }^{18}$ In addition, Mariani et al ${ }^{19}$ determined the $\mathrm{IC}_{50}$ of oxaliplatin in 22 CRC cell lines, which demonstrated a great variability. Specifically, the $\mathrm{IC}_{50}$ of SW116 treated with oxaliplatin was only $0.05 \pm 0.01 \mu \mathrm{M}$, while that of DLD- 1 was up to $65.43 \pm 23.96 \mu \mathrm{M}$. The variability of $\mathrm{IC}_{50}$ in different cell lines is likely due to the heterogeneity of tumor cells, the socalled cell-specific response. After all, each cancer cell line is completely unique.

When examining whether deguelin led to cell apoptosis, we found that protein expression of both Bcl-2 and survivin was decreased following deguelin treatment in CRC cell lines. Survivin is a member of the inhibitor of apoptosis protein (IAP) family, ${ }^{20}$ commonly expressed in cancer cell lines to suppress apoptosis. The Bcl-2 family including over 17 members functions as survival/death switch under stress. ${ }^{21}$ In addition, Bcl-2 was the first identified apoptotic regulator. ${ }^{22}$ In previous studies, targeting Bcl-2 and its family members can be utilized to enhance therapeutic sensitivity in estrogen receptor-positive breast cancer by inducing apoptosis. ${ }^{23}$

It has been reported that multiple factors can affect the timeline of apoptosis events, including the cell lines examined, apoptosis-inducing agents, drug concentration, and exposure times. In our study, deguelin induced apoptosis in SW620, as indicated by flow cytometry. However, the expression of cleaved caspase 3 and cleaved PARP was not obviously altered following deguelin treatment in SW620. In a previous study by Belmokhtar et al, ${ }^{24}$ staurosporine could induce apoptosis through both caspase-dependent and caspase-independent mechanisms in L1210 cell line, which was originally developed in 1949 as a carcinogeninduced acute lymphoid leukemia in DBA mouse strain. Additionally, Pozo-Guisado et $\mathrm{al}^{25}$ reported that resveratrol induced apoptosis through caspase-independent mechanism with the downregulation of Bcl-2 in MCF-7 human breast cancer cell line. Taken together, it is possible that deguelin induced apoptosis in SW620 cells without obvious activation of caspase 3 .

p38 MAPKs are a family of serine/threonine-directed kinases that are typically characterized as stress-activated kinases. ${ }^{15,26}$ Multiple studies have demonstrated the important role of p38 MAPKs in the regulation of cell apoptosis, cell cycle arrest, and growth inhibition. ${ }^{16}$ p38 MAPKs play a dual role in modulating cell apoptosis. On one hand, 
p38 MAPK pathway can mediate apoptosis in response to extracellular stimuli such as chemotherapeutic agents. ${ }^{15,16}$ Anthocyanins, belonging to flavonoids, induced apoptosis in HCT-116 cells via activation of $\mathrm{p} 38$ MAPK. ${ }^{27}$ Cyclophosphamide enhanced MDA-MB-231 cell apoptosis through activating $\mathrm{p} 38$ MAPK pathway. ${ }^{28}$ In addition, the activation of p38 MAPK pathway, at least, partially promoted the chemosensitivity of glioma cell lines to cis-diaminedichloroplatinum..$^{29}$ On the other hand, phosphorylated $\mathrm{p} 38$, significantly increased resistance to docetaxel-induced apoptosis in prostate cancer cells. ${ }^{30} \mathrm{SB} 203580$, an inhibitor of p38 MAPK, sensitized breast and colon cancer cells to cisplatin-induced apoptosis. ${ }^{31}$

\section{Conclusion}

Collectively, our study demonstrates that deguelin, a natural compound, induces apoptosis in CRC cells, partially via activation of the 38 MAPK signaling pathway. Inhibition of p38 MAPK pathway can attenuate deguelin-induced growth inhibition and apoptosis. Therefore, deguelin might serve as a novel complement for CRC treatment, while further studies are warranted for clinical evaluation of deguelin as a single therapeutic agent or in combination with currently used agents for CRC.

\section{Acknowledgments}

This study was funded by grants from the National Natural Science Foundation of China (Nos 81772545 and 81702331), the National Key R\&D Program of China (No 2017YFC0908200), and the Key Technology Research and Development Program of Zhejiang Province (No 2017C03017).

\section{Author contributions}

Kefeng Ding, Qian Xiao, and Ying Yuan conceived and designed the experiments. Liubo Chen, Kai Jiang, Haiyan Chen, and Xinyi Zhou performed the experiments. Yang Tang and Yinuo Tan analyzed the data. Liubo Chen wrote the manuscript. All authors contributed to data analysis, drafting and revising the article, gave final approval of the version to be published, and agree to be accountable for all aspects of the work.

\section{Disclosure}

The authors report no conflicts of interest in this work.

\section{References}

1. Siegel RL, Miller KD, Jemal A. Cancer statistics, 2016. CA Cancer J Clin. 2016;66(1):7-30.

2. Chen W, Zheng R, Baade PD, et al. Cancer statistics in China, 2015. CA Cancer J Clin. 2016;66(2):115-132.
3. Cersosimo RJ. Management of advanced colorectal cancer, Part 1. Am J Health Syst Pharm. 2013;70(5):395-406.

4. Murillo G, Peng X, Torres KE, Mehta RG. Deguelin inhibits growth of breast cancer cells by modulating the expression of key members of the Wnt signaling pathway. Cancer Prev Res (Phila). 2009;2(11):942-950.

5. Lee HY, Oh SH, Woo JK, et al. Chemopreventive effects of deguelin, a novel Akt inhibitor, on tobacco-induced lung tumorigenesis. $J$ Natl Cancer Inst. 2005;97(22):1695-1699.

6. Yan B, Zhao D, Yao Y, Bao Z, Lu G, Zhou J. Deguelin Induces the Apoptosis of Lung Squamous Cell Carcinoma Cells through Regulating the Expression of Galectin-1. Int J Biol Sci. 2016;12(7):850-860.

7. Boreddy SR, Srivastava SK. Deguelin suppresses pancreatic tumor growth and metastasis by inhibiting epithelial-to-mesenchymal transition in an orthotopic model. Oncogene. 2013;32(34):3980-3991.

8. Lee HY, Suh YA, Kosmeder JW, Pezzuto JM, Hong WK, Kurie JM Deguelin-induced inhibition of cyclooxygenase-2 expression in human bronchial epithelial cells. Clin Cancer Res. 2004;10(3):1074-1079.

9. Memmott RM, Dennis PA. The role of the Akt/mTOR pathway in tobacco carcinogen-induced lung tumorigenesis. Clin Cancer Res. 2010;16(1):4-10.

10. Oh SH, Woo JK, Jin Q, et al. Identification of novel antiangiogenic anticancer activities of deguelin targeting hypoxia-inducible factor-1 alpha. Int J Cancer. 2008;122(1):5-14.

11. Suh YA, Kim JH, Sung MA, et al. A novel antitumor activity of deguelin targeting the insulin-like growth factor (IGF) receptor pathway via up-regulation of IGF-binding protein-3 expression in breast cancer. Cancer Lett. 2013;332(1):102-109.

12. Kang HW, Kim JM, Cha MY, Jung HC, Song IS, Kim JS. Deguelin, an Akt inhibitor, down-regulates NF- $\mathrm{kB}$ signaling and induces apoptosis in colon cancer cells and inhibits tumor growth in mice. Dig Dis Sci. 2012;57(11):2873-2882.

13. Jin Q, Menter DG, Mao L, Hong WK, Lee HY. Survivin expression in normal human bronchial epithelial cells: an early and critical step in tumorigenesis induced by tobacco exposure. Carcinogenesis. 2008;29(8):1614-1622.

14. Loo DT. In situ detection of apoptosis by the TUNEL assay: an overview of techniques. Methods Mol Biol. 2011;682:3-13.

15. Olson JM, Hallahan AR. p38 MAP kinase: a convergence point in cancer therapy. Trends Mol Med. 2004;10(3):125-129.

16. Cuadrado A, Nebreda AR. Mechanisms and functions of p38 MAPK signalling. Biochem J. 2010;429(3):403-417.

17. Xia Y, Shen S, Verma IM. NF- $\kappa B$, an active player in human cancers. Cancer Immunol Res. 2014;2(9):823-830.

18. Hsu YC, Chiang JH, Yu CS, et al. Antitumor effects of deguelin on H460 human lung cancer cells in vitro and in vivo: Roles of apoptotic cell death and H460 tumor xenografts model. Environ Toxicol. 2017;32(1):84-98.

19. Mariani M, Zannoni GF, Sioletic S, et al. Gender influences the class III and $\mathrm{V} \beta$-tubulin ability to predict poor outcome in colorectal cancer. Clin Cancer Res. 2012;18(10):2964-2975.

20. Tamm I, Wang Y, Sausville E, et al. IAP-family protein survivin inhibits caspase activity and apoptosis induced by Fas (CD95), Bax, caspases, and anticancer drugs. Cancer Res. 1998;58(23):5315-5320.

21. Cory S, Adams JM. The Bcl2 family: regulators of the cellular life-ordeath switch. Nat Rev Cancer. 2002;2(9):647-656.

22. Adams JM, Cory S. The Bcl-2 apoptotic switch in cancer development and therapy. Oncogene. 2007;26(9):1324-1337.

23. Merino D, Lok SW, Visvader JE, Lindeman GJ. Targeting BCL-2 to enhance vulnerability to therapy in estrogen receptor-positive breast cancer. Oncogene. 2016;35(15):1877-1887.

24. Belmokhtar CA, Hillion J, Ségal-Bendirdjian E. Staurosporine induces apoptosis through both caspase-dependent and caspase-independent mechanisms. Oncogene. 2001;20(26):3354-3362.

25. Pozo-Guisado E, Merino JM, Mulero-Navarro S, et al. Resveratrolinduced apoptosis in MCF-7 human breast cancer cells involves a caspase-independent mechanism with downregulation of Bcl-2 and NF-kappaB. Int J Cancer. 2005;115(1):74-84. 
26. Grossi V, Peserico A, Tezil T, Simone C. p38 $\alpha$ MAPK pathway: a key factor in colorectal cancer therapy and chemoresistance. World J Gastroenterol. 2014;20(29):9744-9758.

27. Shin DY, Lee WS, Lu JN, et al. Induction of apoptosis in human colon cancer HCT-116 cells by anthocyanins through suppression of Akt and activation of p38-MAPK. Int J Oncol. 2009;35(6):1499-504.

28. Pang H, Cai L, Yang Y, Chen X, Sui G, Zhao C. Knockdown of osteopontin chemosensitizes MDA-MB-231 cells to cyclophosphamide by enhancing apoptosis through activating p38 MAPK pathway. Cancer Biother Radiopharm. 2011;26(2):165-173.
29. Lou X, Zhou Q, Yin Y, Zhou C, Shen Y. Inhibition of the met receptor tyrosine kinase signaling enhances the chemosensitivity of glioma cell lines to CDDP through activation of 338 MAPK pathway. Mol Cancer Ther. 2009;8(5):1126-1136.

30. Gan L, Wang J, Xu H, Yang X. Resistance to docetaxel-induced apoptosis in prostate cancer cells by p38/p53/p21 signaling. Prostate. 2011;71(11): 1158-1166.

31. Pereira L, Igea A, Canovas B, Dolado I, Nebreda AR. Inhibition of p38 MAPK sensitizes tumour cells to cisplatin-induced apoptosis mediated by reactive oxygen species and JNK. EMBO Mol Med.2013;5(11):1759-1774. 


\section{Supplementary material}

A sw620

24 hours 0

$\bar{\alpha}$
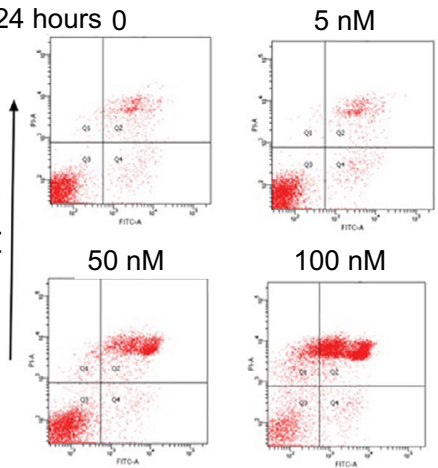

Annexin V

C<smiles>[R]O</smiles>

24 hours 0

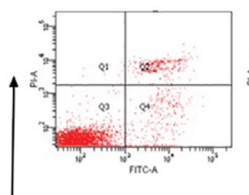

$\bar{\alpha}$

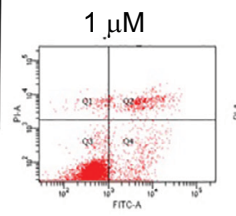

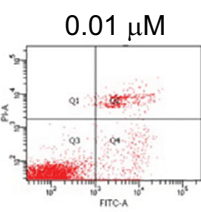
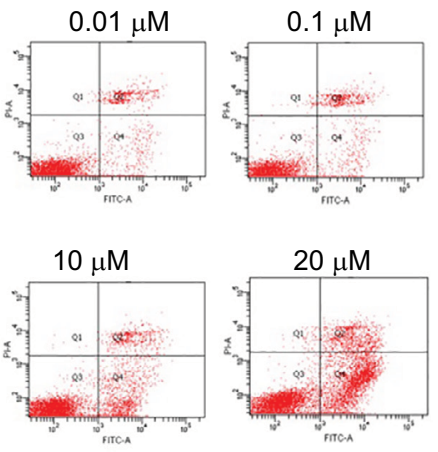

Annexin V

B
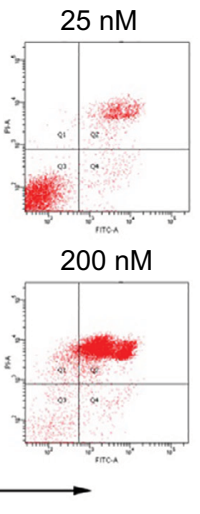

$\bar{\alpha}$

620

48 hours 0
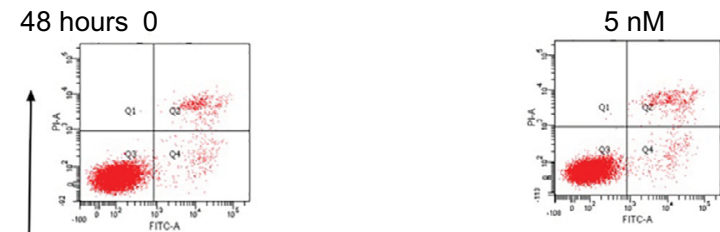

|

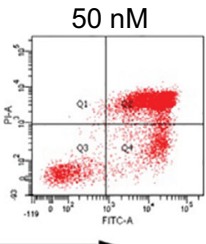

Annexin V

D

RKO

48 hours 0

$\overline{\mathbf{2}}$
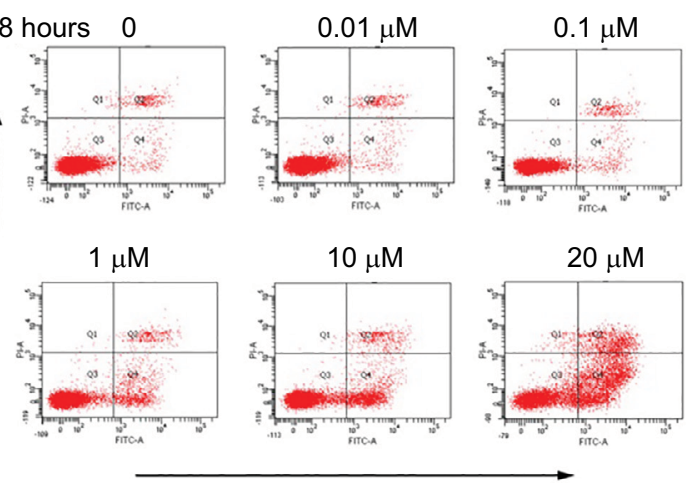

Annexin V

Figure SI Representative images of cell apoptosis induced by deguelin.

Notes: SW620 cells were treated without or with indicated concentrations of deguelin for (A) 24 hours or (B) 48 hours. RKO cells were treated without or with indicated concentrations of deguelin for (C) 24 hours or (D) 48 hours.

Cancer Management and Research

\section{Publish your work in this journal}

Cancer Management and Research is an international, peer-reviewed open access journal focusing on cancer research and the optimal use of preventative and integrated treatment interventions to achieve improved outcomes, enhanced survival and quality of life for the cancer patient. The manuscript management system is completely online and includes

\section{Dovepress}

a very quick and fair peer-review system, which is all easy to use. Visit http://www.dovepress.com/testimonials.php to read real quotes from published authors. 